OPEN ACCESS

Edited by:

Susan Hillier

University of South Australia, Australia

Reviewed by:

Filippo Ghin

Dresden University of Technology,

Germany

Abraham Zangen,

Ben-Gurion University of the Negev,

Israel

Sho Kojima,

Niigata University of Health

and Welfare, Japan

*Correspondence:

Akiyoshi Matsugi

a-matsugi@reha.shijonawate-

gakuen.ac.jp;

aki.pt0422@gmail.com

Specialty section:

This article was submitted to

Perception Science,

a section of the journal

Frontiers in Neuroscience

Received: 18 October 2019

Accepted: 30 March 2020

Published: 28 April 2020

Citation:

Matsugi A, Douchi S, Hasada R,

Mori N, Okada Y, Yoshida N, Nishishita S, Hosomi K and Saitoh Y

(2020) Cerebellar Repetitive

Transcranial Magnetic Stimulation and Noisy Galvanic Vestibular

Stimulation Change Vestibulospinal

Function. Front. Neurosci. 14:388.

doi: 10.3389/fnins.2020.00388

\section{Cerebellar Repetitive Transcranial Magnetic Stimulation and Noisy Galvanic Vestibular Stimulation Change Vestibulospinal Function}

\author{
Akiyoshi Matsugi"*, Shinya Douchi², Rikiya Hasada ${ }^{3}$, Nobuhiko Mori $^{4,5}$, Yohei Okada ${ }^{6,7}$, \\ Naoki Yoshida ${ }^{8,9}$, Satoru Nishishita ${ }^{8,9}$, Koichi Hosomi ${ }^{4,5}$ and Youichi Saito ${ }^{4,5}$ \\ ${ }^{1}$ Faculty of Rehabilitation, Shijonawate Gakuen University, Daito, Japan, ${ }^{2}$ Department of Rehabilitation, National Hospital \\ Organization Kyoto Medical Center, Kyoto, Japan, ${ }^{3}$ Department of Rehabilitation, Nagahara Hospital, Higasiosaka, Japan, \\ ${ }^{4}$ Department of Neuromodulation and Neurosurgery, Graduate School of Medicine, Osaka University, Osaka, Japan, \\ ${ }^{5}$ Department of Neurosurgery, Graduate School of Medicine, Osaka University, Osaka, Japan, ${ }^{6}$ Faculty of Health Sciences, \\ Kio University, Koryo, Japan, ${ }^{7}$ Neurorehabilitation Research Center, Kio University, Koryo, Japan, ${ }^{8}$ Institute of Rehabilitation \\ Science, Tokuyukai Medical Corporation, Toyonaka, Japan, ${ }^{9}$ Kansai Rehabilitation Hospital, Toyonaka, Japan
}

Background: The cerebellum strongly contributes to vestibulospinal function, and the modulation of vestibulospinal function is important for rehabilitation. As transcranial magnetic stimulation (TMS) and electrical stimulation may induce functional changes in neural systems, we investigated whether cerebellar repetitive TMS (crTMS) and noisy galvanic vestibular stimulation (nGVS) could modulate vestibulospinal response excitability. We also sought to determine whether crTMS could influence the effect of nGVS.

Methods: Fifty-nine healthy adults were recruited; 28 were randomly allocated to a real-crTMS group and 31 to a sham-crTMS group. The crTMS was conducted using 900 pulses at $1 \mathrm{~Hz}$, while the participants were in a static position. After the crTMS, each participant was allocated to either a real-nGVS group or sham-nGVS group, and nGVS was delivered (15 min., $1 \mathrm{~mA} ; 0.1-640 \mathrm{~Hz}$ ) while patients were in a static position. The H-reflex ratio (with/without bilateral bipolar square wave pulse GVS), which reflects vestibulospinal excitability, was measured at pre-crTMS, post-crTMS, and post-nGVS.

Results: We found that crTMS alone and nGVS alone have no effect on $\mathrm{H}$-reflex ratio but that the effect of nGVS was obtained after crTMS.

Conclusion: crTMS and nGVS appear to act as neuromodulators of vestibulospinal function.

Keywords: cerebellum, transcranial magnetic stimulation, $\mathrm{H}$-reflex, vestibular, galvanic vestibular stimulation

\section{INTRODUCTION}

The vestibular system and cerebellum allow for postural control and adaptation to several physical environments in human daily life. The investigation of the function of vestibular, cerebellum, and functional connectivity of both is important for the improvement of rehabilitation protocols. Studies using electrical stimulation and magnetic stimulation have revealed these functions. 
Electrical stimulation to deep cerebellar nuclei induces excitatory and inhibitory postsynaptic potentials in vestibular neurons through polysynaptic pathways (Ito et al., 1970), and lesions in the cerebellum disturb long-term adaptive changes in vestibular reflexes in animal models (Miles and Eighmy, 1980; Ito, 1998). These findings provide evidence for the functional connectivity between the cerebellum and vestibular complex (Jang et al., 2018). This connectivity is established through avenues such as the fastigial nucleus and interposed and dentate nuclei (Delfini et al., 2000). Such connectivity also refers to cerebellar involvement in the modulation of the excitability of vestibular reflexes (Straka et al., 2016).

Transcranial magnetic stimulation (TMS) non-invasively induces action potentials in cortical neurons and inhibits contralateral corticospinal excitability when applied over the cerebellar hemisphere (Ugawa et al., 1995; Daskalakis et al., 2004; Grimaldi et al., 2014; Matsugi and Okada, 2017, 2020). Repetitive TMS (rTMS) applied over a cerebellar hemisphere changes cerebellar brain inhibition (CBI) (Popa et al., 2010), indicating that cerebellar TMS (crTMS) can stimulate certain cerebellar tissues and that crTMS can modulate the excitability of cerebellar outputs. TMS applied over the inion improves eye-head coordination (Nagel and Zangemeister, 2003); as this effect requires vestibule-ocular function, the aforementioned finding indicates that TMS applied over the medial cerebellum affects vestibule-ocular function. Furthermore, as the vestibular nuclei comprise the common center for both vestibulo-ocular and vestibulospinal function (Straka et al., 2016), cerebellar TMS may affect vestibulospinal function. Therefore, in this study, we used the rTMS over inion to stimulate the central cerebellum, investigating whether cerebellar stimulation affect the vestibulospinal function (first aim of this study).

To test vestibulospinal function, galvanic vestibular stimulation (GVS) can be used (Fitzpatrick and Day, 2004). The firing rate of primary vestibular afferents can be decreased by the anodal square-wave pulse GVS (sqGVS) and increased by the cathodal sqGVS (Kim and Curthoys, 2004). Direct recording demonstrates that this stimulation induces action potentials in the vestibulospinal tract of the spinal cord and motor responses in target muscles with short latency (Muto et al., 1995). Furthermore, sqGVS can induce body sway in standing individuals (Fitzpatrick et al., 1994; Day et al., 2002). Changes in the activities of muscles that maintain postural control can be measured by electromyography (EMG) and the Hoffman reflex (H-reflex) (Iles and Pisini, 1992; Britton et al., 1993; Ali et al., 2003; Matsugi et al., 2017; Matsugi, 2019), which reflect the excitability of the spinal motoneuron pool (Knikou, 2008). Applied to an individual in a static position unaffected by natural body sway, change in the range of joints, or background EMG activity, sqGVS modulates the excitability of the H-reflex in the soleus muscle (Kennedy and Inglis, 2001; Ghanim et al., 2009; Lowrey and Bent, 2009; Okada et al., 2018). These observations indicate that the H-reflex-modulation induced by sqGVS reflects changes in the excitability of the vestibulospinal response. Therefore, in this study, we used this method to test vestibulospinal function.
Galvanic vestibular stimulation is often used not only to test vestibulospinal function but also to improve it. To improve balance mediated via facilitation of vestibulospinal function, the square-wave pulse GVS has not been used and random noise GVS (nGVS) is often used recently. The nGVS reportedly improves body balance in adults irrespective of age as well as in patients with vestibular disorder (Wuehr et al., 2017; Fujimoto et al., 2018). The stochastic resonance of noise addition to nonlinear systems inducing the change in plasticity of information processing in neural systems may change the threshold or excitability of the motor response by vestibular input (McDonnell and Ward, 2011). However, it is unclear whether nGVS induces changes in vestibulospinal response excitability. Therefore, we investigated whether nGVS modulates the vestibulospinal function, as estimated by the H-reflex-modulation induced by sqGVS (second aim of this study).

Furthermore, the cerebellum is involved in the plasticity of vestibular reflex excitability, because crTMS modulates the effect of intervention for increased vestibulo-ocular movement for dynamic gaze (Matsugi et al., 2019). Deep cerebellar nuclei and Purkinje fibers in the cerebellar gray matter project to the vestibular nucleus. Stimulation of the cerebellar surface induces postsynaptic inhibitory or excitatory postsynaptic potentials in vestibular nuclei (Ito et al., 1970). Low-frequency repetitive stimulation of cortical neurons induces the long-term depression of synaptic excitability (Huerta and Volpe, 2009). Therefore, we hypothesized that crTMS affects the vestibular modulation via interventions such as nGVS in addition to that of exercise. Therefore, as the third aim of this study, we investigated whether rTMS applied over the cerebellum influenced the effect of nGVS on vestibulospinal excitability.

In summary, in this study, we investigated whether crTMS alone and nGVS alone modulate vestibulospinal function estimated by the H-reflex modulation induced by sqGVS. Further, we investigated whether crTMS modulates the effect of nGVS on the H-reflex-modulation induced by sqGVS.

\section{MATERIALS AND METHODS}

\section{Participants}

Fifty-nine healthy adults (mean age, $23.8 \pm 4.5$ years; 40 men) participated in this study. None of the participants had histories of epilepsy or other neurological diseases. The Ethics Committee of Shijonawate Gakuen University approved the experimental procedures (approval code: 29-4), and this study was conducted according to the principles and guidelines of the Declaration of Helsinki; written informed consent was obtained from all participants.

\section{General Procedure}

This study was conducted with a sham-controlled, double-blind design. The crTMS and nGVS conditions were blinded for participants and assessors when the assessments of vestibulospinal response were performed. Figure 1 presents the general procedures. All participants were allocated to either the sham-crTMS $(n=31)$ or real-crTMS groups $(n=28)$. After 


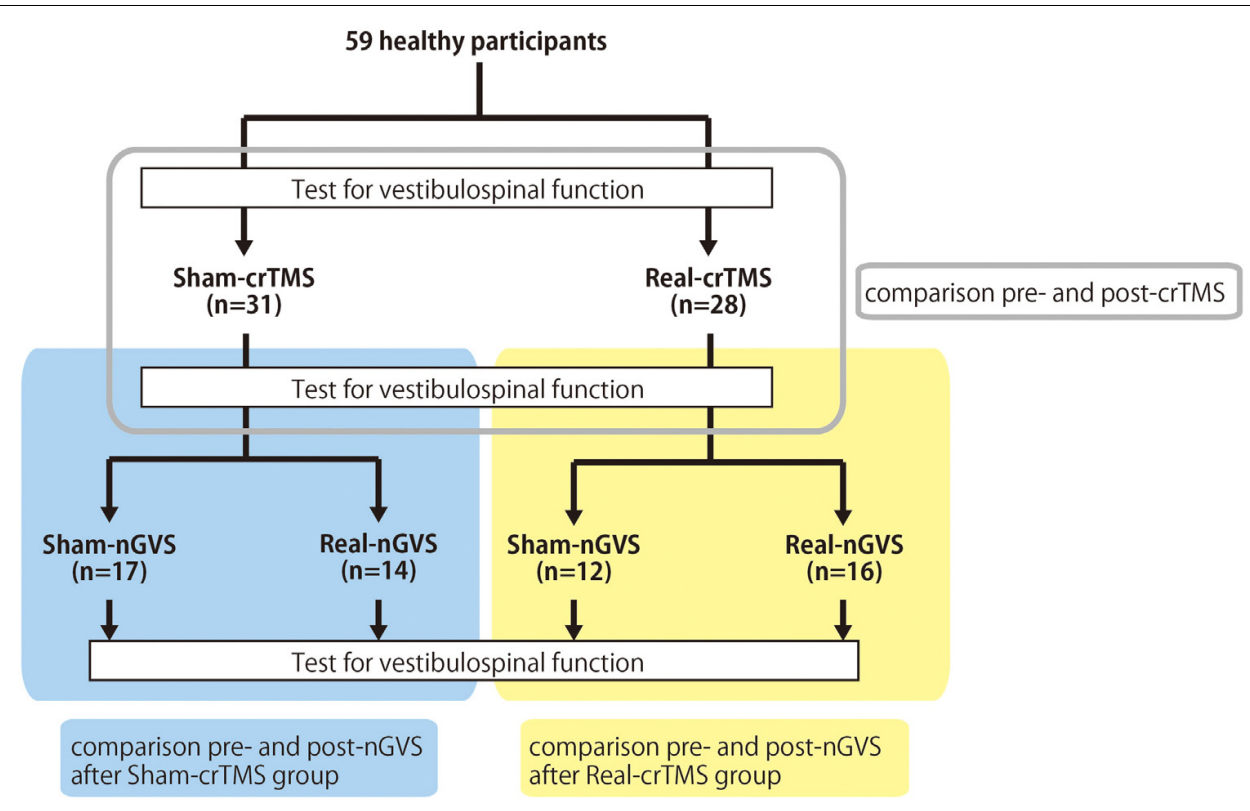

FIGURE 1 | Experimental procedure and analysis. Fifty-nine healthy participants were allocated to either the sham-cerebellar repetitive TMS (crTMS) or real-crTMS groups. After crTMS, the participants were further allocated to either sham-noisy galvanic vestibular stimulation (nGVS) or real-nGVS groups. Tests for vestibulospinal function, including the H-reflex with/without conditioning square-wave pulse GVS, were conducted pre-crTMS, post-crTMS, and post-nGVS. In Analysis 1, the test parameters were compared between pre- and post-crTMS to elucidate the effect of crTMS on vestibulospinal function. In Analysis 2 , the test parameters were compared between pre- and post-nGVS (post-sham-crTMS) to assess the effect of nGVS on vestibulospinal function. In Analysis 3 , the test parameters were compared between pre- and post-nGVS (post-real-crTMS) to gauge the influence of crTMS on the effect of nGVS on vestibulospinal function. crTMS, repetitive transcranial magnetic stimulation; nGVS, noisy galvanic vestibular stimulation.

sham- or real-crTMS was completed, the participants in both groups were further subdivided into sham-nGVS and real-nGVS groups (after sham-crTMS: $n=17$ and $n=14$, respectively; after real-crTMS: $n=12$ and $n=16$, respectively), and then nGVS were conducted. Hence, all participants were randomly assigned to one of four groups, and three assessments of vestibulospinal function were conducted before (1st) and after crTMS (2nd) and after nGVS (3rd). If the participant experienced the sensation of the nGVS or could not endure the sqGVS in the test stimulation, the examination was ceased immediately.

Before the examination, we confirmed that the sqGVS did not produce sensations of pain or phosphine behind the eyes but did prompt body sway to the anodal side in participants standing with their eyes closed, feet together, and head facing forward (Fitzpatrick and Day, 2004) to test whether they were responders or non-responders to square-wave pulse GVS. In this timing, if the participant cannot endure square-wave pulses at $3 \mathrm{~mA}$ using all tests for vestibulospinal function, the participant did not participate in subsequent experiments. No participant responded to sqGVS at $3 \mathrm{~mA}$, but four participants were excluded owing to the existence of unbearable pain (see "Results" section). We asked participants to report any sensation in response to nGVS; participants reporting sensation were excluded from analysis.

During the tests for vestibulospinal function (1st, 2nd, and 3 rd), crTMS (sham or real) and nGVS (sham or real), participants lay down in the prone position while relaxing on the bed. The experiments were performed in the following order: (1) test for vestibulospinal function (1st test), (2) sham- or real-crTMS,
(3) test for vestibulospinal function (2nd test), (4) sham- or real-nGVS, and (5) test for vestibulospinal function (3rd test).

\section{Conditioning Stimulation Cerebellar Repetitive TMS (crTMS)}

The participants were instructed to lie down on a bed in the prone position. Because previous studies have shown that the figure-of-eight coil could stimulate the cerebellum (Haarmeier and Kammer, 2010; Popa et al., 2010; Tremblay et al., 2016), a magnetic stimulator (MagPro compact, MagVenture, Denmark) was used to deliver TMS to the cerebellum with a butterfly coil (MC-B70, MagVenture, Denmark) (Matsugi et al., 2019). The center of the coil's junction was set at a distance $1 \mathrm{~cm}$ below the inion to stimulate the central region of the cerebellum (Zangemeister and Nagel, 2001; Nagel and Zangemeister, 2003; Jayasekeran et al., 2011; Hardwick et al., 2014; van Dun et al., 2017); prior research has demonstrated that stimulation from this position can modulate vestibular and ocular motor functions (Zangemeister and Nagel, 2001; Nagel and Zangemeister, 2003; Jenkinson and Miall, 2010). As previous studies have observed that an upward current applied to the cerebellum can effectively stimulate this region (Ugawa et al., 1995; Hiraoka et al., 2010; Matsugi et al., 2013), the coil was oriented such that the current therein was directed downward to deliver the upward current to the brain (van Dun et al., 2017). TMS intensity was set to $50 \%$ of the maximum stimulator output: the same setting as those employed by previous studies investigating cerebellar and vestibular functions (Zangemeister and Nagel, 


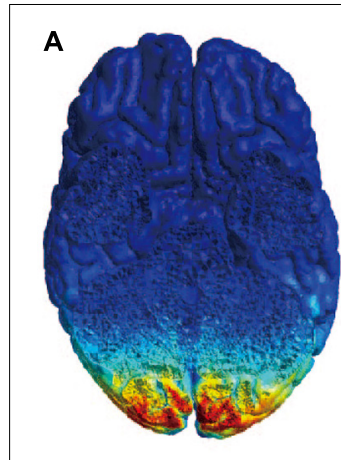

0

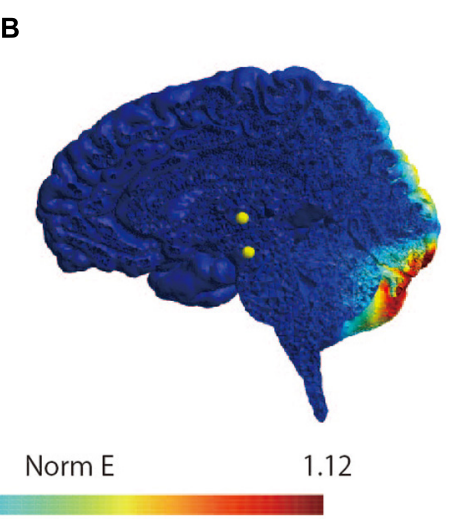

FIGURE 2 | Simulation of the electrical field induced by cerebellar TMS. Simulation of the electrical field induced by TMS with butterfly coil using SimNIBS in horizontal slice (A) and sagittal slice (B). The scale represents the magnitude of the electric field (Volts/meter) induced by TMS over the site at $1 \mathrm{~cm}$ under the inion.

2001; Nagel and Zangemeister, 2003; Haarmeier and Kammer, 2010; Jenkinson and Miall, 2010; van Dun et al., 2017; Matsugi et al., 2019). Because a $1-\mathrm{Hz}$ crTMS reduces motor function and motor adaptation (Miall and Christensen, 2004; Jenkinson and Miall, 2010) the inter-stimulus interval was set at $1 \mathrm{~s}$, and 900 pulses were applied (Fierro et al., 2007; Popa et al., 2010; Matsugi et al., 2019). In the stimulation condition, electrical field stimulation of the brain structures was performed with SimNIBS software (version 2.1.1) using default head models, biological tissue conductivity values included in the software, and the aforementioned parameters of the TMS using a butterfly coil (shown in Figure 2) (Thielscher et al., 2015). The coil was held at a $90^{\circ}$ angle from the scalp over the inion when delivering sham-TMS (Hiraoka et al., 2010; Matsugi et al., 2013, 2014, 2019).

\section{Noisy Galvanic Vestibular Stimulation (nGVS)}

Noisy galvanic vestibular stimulation was performed as previously reported (Inukai et al., 2018). nGVS was delivered via $\mathrm{Ag} / \mathrm{AgCl}$ surface electrodes (Blue Sensor EKG Snap Electrode, overall dimensions: $48 \mathrm{~mm} \times 57 \mathrm{~mm}$, Ambu, Baltorpbakken, Denmark) affixed to the right and left mastoid processes. A DCSTIMULATOR PLUS (Eldith, NeuroConn GmbH, Ilmenau, Germany) was used to deliver random noise galvanic stimulation to the primary vestibular nerve. For nGVS in the stimulation mode, "noise" was used, a random level of current was generated for every sample (sample rate, 1280 samples/s) (Moliadze et al., 2012; Inukai et al., 2018), and the intensity was set at $1 \mathrm{~mA}$. Statistically, the random numbers were normally distributed over time, the probability density followed a Gaussian bell curve, and all coefficients featured a similar size in the frequency spectrum of this mode. A waveform was applied with $99 \%$ of the values between -0.5 and $+0.5 \mathrm{~mA}$, and only $1 \%$ of the current level was within $\pm 0.51 \mathrm{~mA}$. The stimulation time was set to $900 \mathrm{~s}$, and the current was ramped up and down from $6 \mathrm{~s}$ before the stimulation to $6 \mathrm{~s}$ after its completion (Figure 3). Even if a slight sensation was felt, the participant was excluded from the

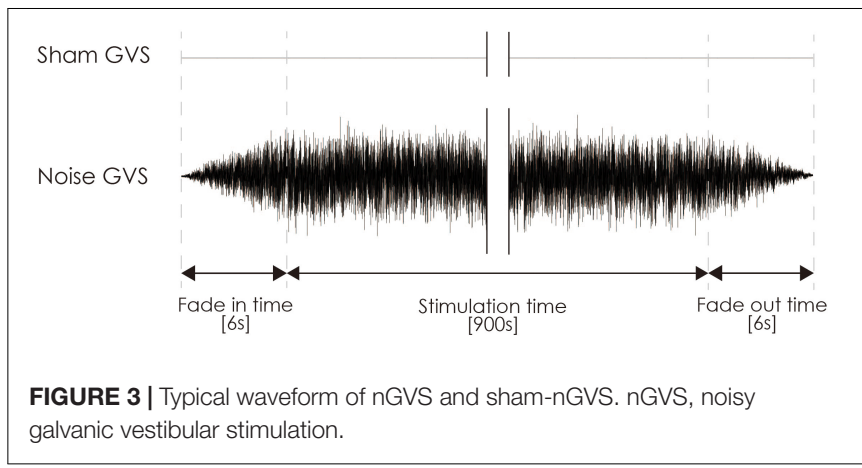

experiment on account of the condition no longer being blind. For sham stimulation, direct current stimulation was applied at an intensity of $0 \mathrm{~mA}$ (sham-nGVS). If the participant sensed the stimulation of the real- or sham-nGVS, the participant was disqualified from further testing.

\section{Test for Vestibulospinal Function}

To estimate the excitability of the vestibulospinal response, the H-reflex during short duration square-wave pulse GVS was measured (Kennedy and Inglis, 2001, 2002; Ghanim et al., 2009; Lowrey and Bent, 2009; Matsugi et al., 2017) before and after crTMS and after nGVS (see Figure 1). The H-reflex indicates the excitability of the spinal motoneuron pool (Knikou, 2008). Short duration square-wave pulse GVS can alter the firing rate of primary vestibular neurons in a polarity-dependent manner (Goldberg et al., 1984), indicating that electrostimulation of the mastoid processes provides constant stimulation to vestibular neurons. Therefore, the modulation of the H-reflex by short duration square-wave pulse GVS reflects the excitability of the vestibulospinal response.

The participant lay down on a bed in the prone position with his or her eyes closed, right and left ankle joints fixed at 90 degrees, and with braces to prevent unwanted movement of ankle joints. A bipolar binaural square-wave pulse GVS was delivered via $\mathrm{Ag} / \mathrm{AgCl}$ surface electrodes affixed to the mastoid processes (Britton et al., 1993; Fitzpatrick et al., 1994; Welgampola and Colebatch, 2001; Ghanim et al., 2009; Lowrey and Bent, 2009; Matsugi et al., 2017) (right, cathode; left, anode; Figure 4). The GVS consisted of a 200-ms square-wave pulse that was delivered using an electrical isolator (SS-104J, Nihon Kohden, Japan) driven by a stimulator (SEN-3301, Nihon Kohden, Japan); the intensity was set at $3 \mathrm{~mA}$ (Fitzpatrick and Day, 2004; Okada et al., 2018).

Electromyography signals used to measure the H-reflex were recorded as previously described (Matsugi et al., 2017). Two $\mathrm{Ag} / \mathrm{AgCl}$ surface-recording electrodes were placed $2 \mathrm{~cm}$ apart on the right soleus muscle. The EMG signals were amplified using an amplifier (MEG-1200, Nihon Kohden, Japan) with a pass-band filter of $15 \mathrm{~Hz}$ to $3 \mathrm{kHz}$. The EMG signals were converted to digital signals at a sampling rate of $10 \mathrm{kHz}$ using an A/D converter (PowerLab 800S, AD Instruments; AD Instruments, Colorado Springs, CO, United States). The digital signals were then stored on a personal computer. 

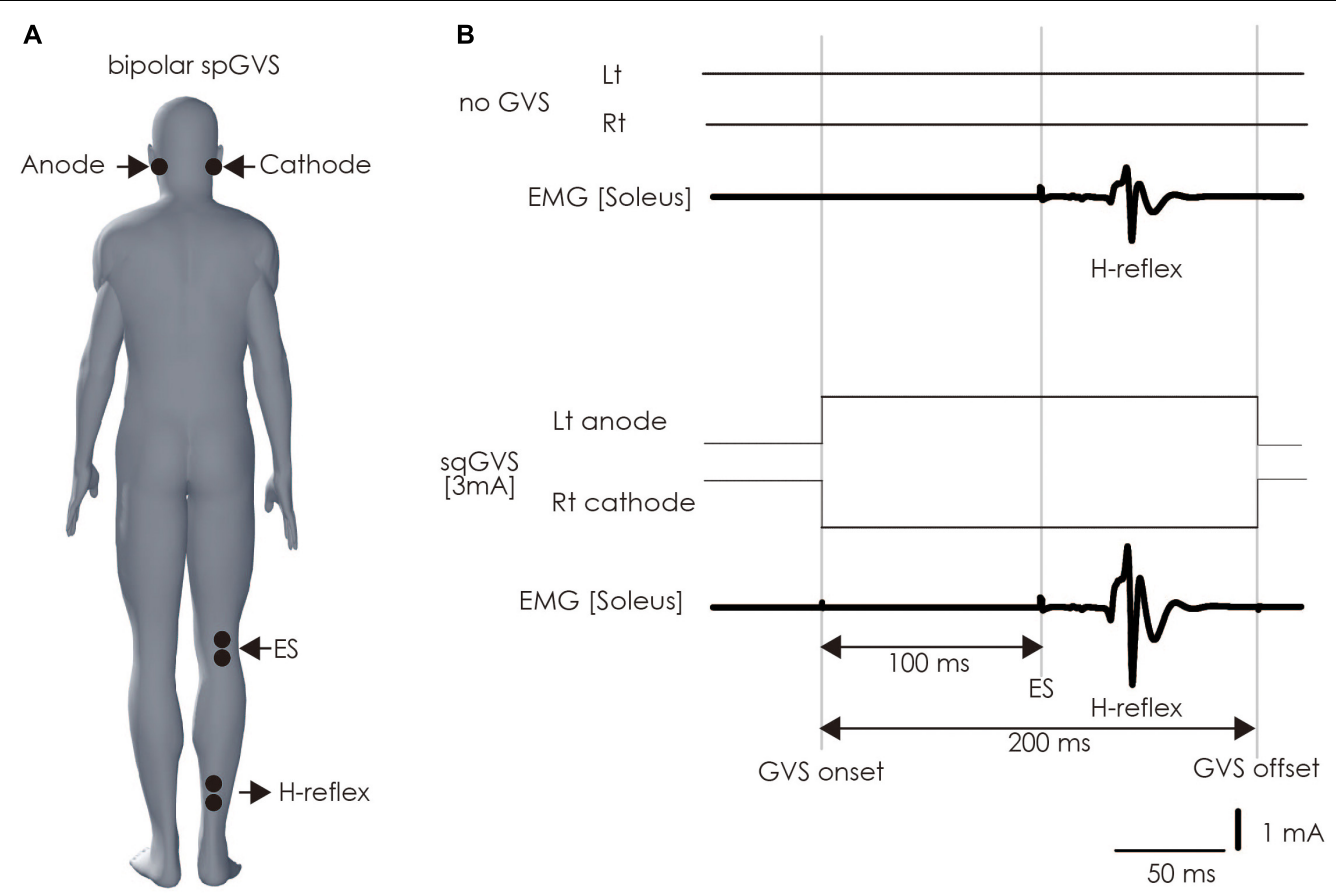

FIGURE 4 | Method of assessing vestibulospinal function. (A) Electrode positioning. The electrode for the GVS was placed on bilateral mastoid processes. The electrodes were placed on the right soleus muscle to stimulate the tibial nerve to induce H-reflex therein and perform electromyography. (B) The sqGVS and typical $\mathrm{H}$-reflex waveforms. The ES to induce the H-reflex was delivered 100 ms before sqGVS onset. GVS, galvanic vestibular stimulation; sqGVS, square-wave pulse galvanic vestibular stimulation; EMG, electromyography; ES, electrical stimulation.

We delivered electrical stimulation (ES) to the right tibial nerve to evoke the H-reflex in the right soleus muscle $100 \mathrm{~ms}$ after the onset of the short duration square-wave pulse GVS (Kennedy and Inglis, 2001; Ghanim et al., 2009; Matsugi et al., 2017) (Figure 4). The H-reflex is reportedly facilitated by cathodal GVS in the inter-stimulus interval (Ghanim et al., 2009). The maximal M-wave (M-max) was measured at the beginning of all of the experimental trials, and the test for the right soleus H-reflex amplitude was periodically adjusted to a level $15-25 \%$ of the $\mathrm{M}$-max during the experiment to adjust for the ascending limb of the H-reflex recruitment curve (Crone et al., 1990; Matsugi et al., 2017). Ten H-reflexes were elicited and recorded in the non-GVS condition (as control). In the right cathodal sqGVS condition, the two trials were performed in a random order, and the interval between tests was set to $>7 \mathrm{~s}$.

Before the test, we confirmed that the sqGVS did not produce sensations of pain or phosphine behind the eyes but did prompt body sway to the anodal side in participants standing with their eyes closed, feet together, and head facing forward (Fitzpatrick and Day, 2004). Furthermore, the GVS response test was conducted more than four times to ensure the participants were habituated to the sqGVS before test trials; although the first GVS responses were larger than the fifth, there was no subsequent change after the fifth trial (Balter et al., 2004).

\section{Analysis}

$\mathrm{H}$-reflex amplitude and $\mathrm{M}$-wave amplitude in individual wave form was measured, and H-reflex as a percent of M-max amplitude was calculated in all trials and examinations based on the formula: H-reflex amplitude/M-max amplitude $\times 100$. M-wave a percent of M-max amplitude was similarly calculated: M-wave amplitude/M-max amplitude $\times 100$. To estimate the excitability of vestibulospinal function, the $\mathrm{H}$-reflex ratio was calculated as the conditioned H-reflex amplitude/unconditioned H-reflex amplitude (Matsugi et al., 2017; Matsugi and Okada, 2020).

To test the baseline stimulation is equal, the paired sample test was conducted. If test of normality (Shapiro-Wilk test) revealed that normality of data, $t$-test was used. If in not normality of data, Wilcoxon test was used.

To test the effect of intervention of crTMS or nGVS (Sham and Real) and time (Pre- and Post-stimulation) on the H-reflex ratio, two-way analysis of variance (TW-ANOVA) was used if equality of variances was confirmed by Levene's test. When a main effect was observed on these parameters, post hoc comparison ( $t$-test) was conducted. When an interaction effect was observed on the means of H-reflex ratios, the post hoc comparison ( $t$-test) was conducted to detect significant differences between groups.

In Analysis 1, to estimate the effect of crTMS on excitability of the vestibulospinal response, the H-reflex ratios obtained from pre-crTMS and post-crTMS in the real- and sham-crTMS conditions were analyzed (Figure 1). In Analysis 2, to gauge the effect of nGVS on the excitability of the vestibulospinal response, the H-reflex ratios obtained from the four pre- and post-nGVS trials performed after the sham-crTMS were analyzed 
TABLE 1 | Paired Samples T-Test (unconditioned M-wave).

\begin{tabular}{|c|c|c|c|c|c|c|c|c|c|c|c|}
\hline & & \multicolumn{2}{|c|}{$\begin{array}{l}\text { Test of normality } \\
\text { (Shapiro-Wilk) }\end{array}$} & \multirow[b]{2}{*}{ Test } & \multirow[b]{2}{*}{ Statistic } & \multirow[b]{2}{*}{ df } & \multirow[b]{2}{*}{$p$} & \multirow[b]{2}{*}{ VS-MPR ${ }^{\star}$} & \multirow[b]{2}{*}{ Effect Size } & \multicolumn{2}{|c|}{$\begin{array}{l}95 \% \mathrm{Cl} \text { for } \\
\text { effect size }\end{array}$} \\
\hline & & $\mathbf{w}$ & $\mathbf{p}$ & & & & & & & Lower & Upper \\
\hline \multirow[t]{4}{*}{ Analysis 1} & Sham & 0.942 & 0.091 & Student & 1.939 & 30 & 0.062 & 2.135 & 0.348 & -0.017 & 0.708 \\
\hline & & & & Wilcoxon & 322 & & 0.152 & 1.286 & 0.298 & -0.095 & 0.611 \\
\hline & Real & 0.708 & $<0.001$ & Student & -1.259 & 27 & 0.219 & 1.107 & -0.238 & -0.612 & 0.14 \\
\hline & & & & Wilcoxon & 177 & & 0.782 & 1 & -0.128 & -0.503 & 0.287 \\
\hline \multirow[t]{4}{*}{ Analysis 2} & Sham & 0.931 & 0.228 & Student & -1.552 & 16 & 0.14 & 1.335 & -0.376 & -0.864 & 0.122 \\
\hline & & & & Wilcoxon & 56 & & 0.353 & 1.001 & -0.268 & -0.673 & 0.26 \\
\hline & Real & 0.836 & 0.014 & Student & 0.458 & 13 & 0.655 & 1 & 0.122 & -0.406 & 0.646 \\
\hline & & & & Wilcoxon & 62 & & 0.583 & 1 & 0.181 & -0.39 & 0.651 \\
\hline \multirow[t]{4}{*}{ Analysis 3} & Sham & 0.7 & $<0.001$ & Student & -1.215 & 11 & 0.25 & 1.062 & -0.351 & -0.927 & 0.241 \\
\hline & & & & Wilcoxon & 30 & & 0.519 & 1 & -0.231 & -0.704 & 0.385 \\
\hline & Real & 0.818 & 0.005 & Student & 1.045 & 15 & 0.313 & 1.012 & 0.261 & -0.242 & 0.756 \\
\hline & & & & Wilcoxon & 84 & & 0.433 & 1 & 0.235 & -0.307 & 0.662 \\
\hline
\end{tabular}

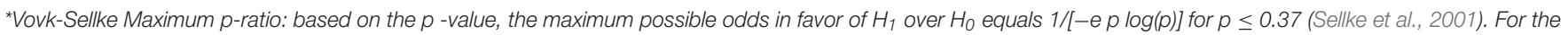

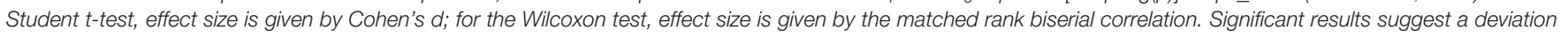
from normality inTest of Normality (Shapiro-Wilk). Bolded values/terms indicate accepted results.

TABLE 2 | Paired samples T-Test (unconditioned H-reflex).

\begin{tabular}{|c|c|c|c|c|c|c|c|c|c|c|c|}
\hline & & \multicolumn{2}{|c|}{$\begin{array}{l}\text { Test of normality } \\
\text { (Shapiro-Wilk) }\end{array}$} & \multirow[b]{2}{*}{ Test } & \multirow[b]{2}{*}{ Statistic } & \multirow[b]{2}{*}{ df } & \multirow[b]{2}{*}{$p$} & \multirow[b]{2}{*}{ VS-MPR ${ }^{\star}$} & \multirow[b]{2}{*}{ Effect Size } & \multicolumn{2}{|c|}{$\begin{array}{l}95 \% \mathrm{Cl} \text { for } \\
\text { effect size }\end{array}$} \\
\hline & & w & $p$ & & & & & & & Lower & Upper \\
\hline \multirow[t]{4}{*}{ Analysis 1} & Sham & 0.975 & 0.652 & Student & -1.547 & 30 & 0.132 & 1.374 & -0.278 & -0.634 & 0.083 \\
\hline & & & & Wilcoxon & 150 & & 0.056 & 2.278 & -0.395 & -0.676 & -0.015 \\
\hline & Real & 0.958 & 0.318 & Student & -0.408 & 27 & 0.687 & 1 & -0.077 & -0.447 & 0.295 \\
\hline & & & & Wilcoxon & 194 & & 0.849 & 1 & -0.044 & -0.437 & 0.362 \\
\hline \multirow[t]{4}{*}{ Analysis 2} & Sham & 0.969 & 0.8 & Student & -0.011 & 16 & 0.992 & 1 & -0.003 & -0.478 & 0.473 \\
\hline & & & & Wilcoxon & 80 & & 0.89 & 1 & 0.046 & -0.459 & 0.528 \\
\hline & Real & 0.563 & $<0.001$ & Student & 1.226 & 13 & 0.242 & 1.071 & 0.328 & -0.217 & 0.86 \\
\hline & & & & Wilcoxon & 74 & & 0.194 & 1.157 & 0.41 & -0.158 & 0.774 \\
\hline \multirow[t]{4}{*}{ Analysis 3} & Sham & 0.92 & 0.285 & Student & 1.34 & 11 & 0.207 & 1.128 & 0.387 & -0.209 & 0.967 \\
\hline & & & & Wilcoxon & 51 & & 0.38 & 1 & 0.308 & -0.312 & 0.744 \\
\hline & Real & 0.941 & 0.36 & Student & 0.054 & 15 & 0.958 & 1 & 0.013 & -0.477 & 0.503 \\
\hline & & & & Wilcoxon & 66 & & 0.94 & 1 & -0.029 & -0.528 & 0.484 \\
\hline
\end{tabular}

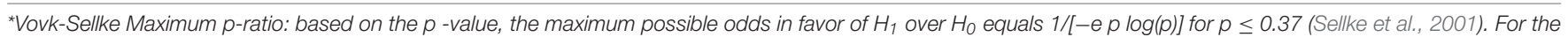

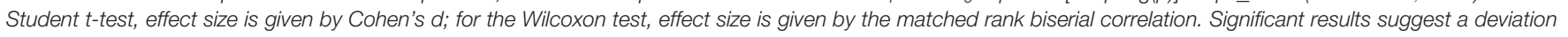
from normality inTest of Normality (Shapiro-Wilk). Bolded values/terms indicate accepted results.

(Figure 1). In analysis 3, to estimate the effect of cerebellar crTMS on the effect of nGVS, the H-reflex ratios obtained from the pre- and post-nGVS trials performed after the real-crTMS were analyzed (Figure 1).

The alpha level was set at 0.05 in all statistical analyses. Statistical analyses were conducted with using $\mathrm{R}$ software (version 3.1.2; the $\mathrm{R}$ Foundation for Statistical Computing, Vienna, Austria).

Post hoc power analysis was conducted to estimate the power ( 1 - beta error probability) for conducting Wilcoxon signed rank sum tests to compare the smallest groups (sham-nGVS in postreal-crTMS, $n=12$ ) with software G* power 3.1 (Version 3.1.9.4) provided by Faul et al. (2007).

\section{RESULTS}

None of the participants experienced any harmful side effects attributable to any of the examinations. As four participants were unable to endure the sqGVS before the examination, the examinations were terminated for these participants. Fifty-nine participants responded to the sqGVS while standing by engaging in a body sway to the anodal side (Fitzpatrick and Day, 2004), and no participant reported to sensation to nGVS.

Tables 1, 2 show the results of Shapiro-Wilk test, and paired sample test in $\mathrm{M}$-wave and unconditioned $\mathrm{H}$-reflex amplitude (Figures 5, 6). These results indicate the there was no significant difference between stimulation conditioned. 

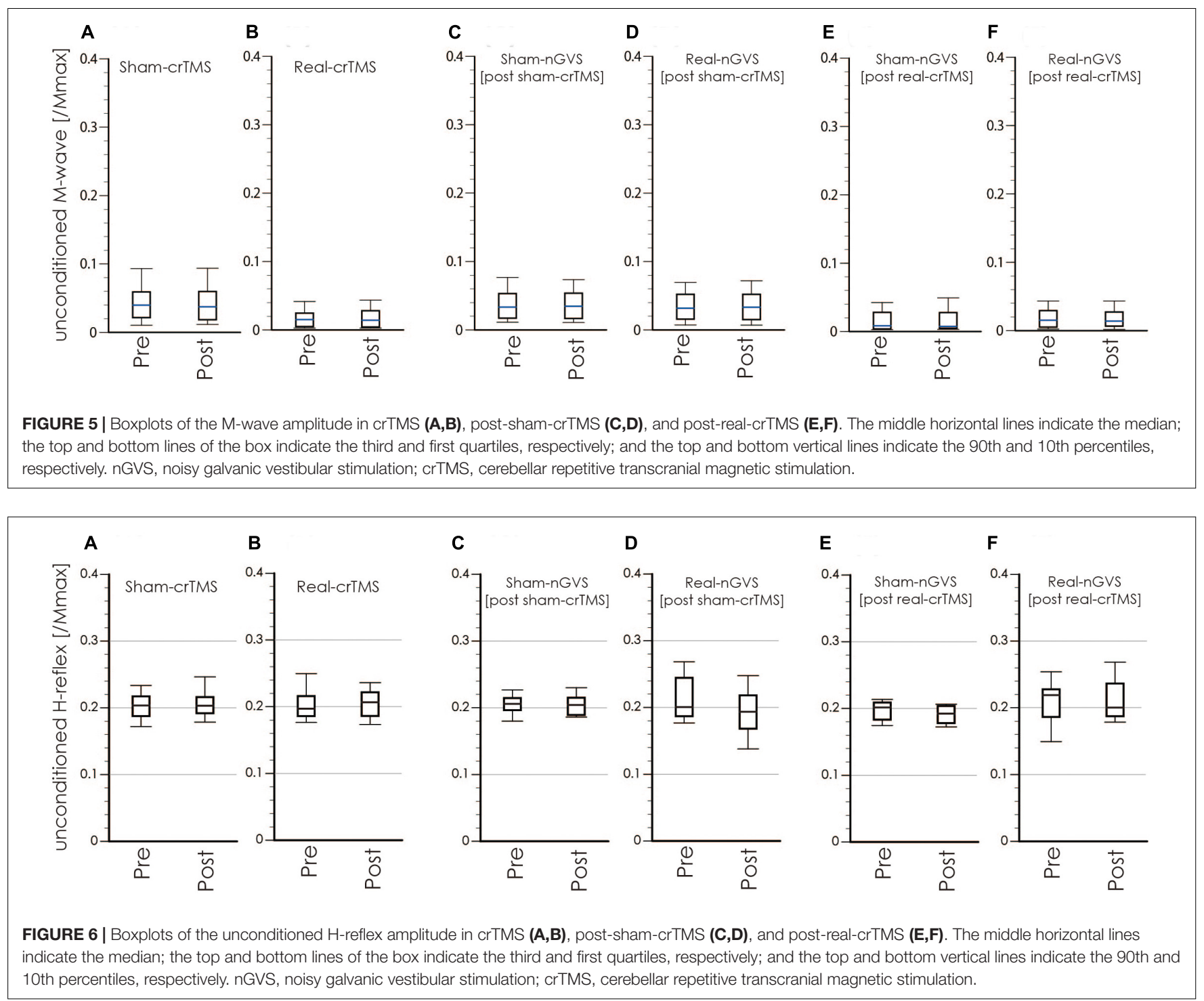

Table 3 shows result of Levene's test, and this test revealed that all parameters had equal variances for TW-ANOVA. These results indicate that there were equality of data and we can accept the result of parametric TW-ANOVA. Table 4 shows the For the H-reflex ratio in analyses 1 and 2, there was no significant effect of intervention and time, but in analysis 3 , there was a significant main effect of intervention and no significant effect of time on the H-reflex ratio (Figure 7).

TABLE 3 | Test for equality of variances (Levene's) for two-way ANOVA.

\begin{tabular}{lccccc}
\hline & $\boldsymbol{F}$ & df1 & df2 & $\boldsymbol{p}$ & VS-MPR $^{*}$ \\
\hline Analysis 1 & 2 & 3 & 114 & 0.118 & 1.459 \\
Analysis 2 & 1.428 & 3 & 58 & 0.244 & 1.069 \\
Analysis 3 & 0.967 & 3 & 52 & 0.415 & 1
\end{tabular}

*Vovk-Sellke maximum p-ratio: based on the $p$-value, the maximum possible odds in favor of $H_{1}$ over $H_{0}$ equals $1 /[-e p \log (p)]$ for $p \leq 0.37$ (Sellke et al., 2001).
The post hoc power analysis revealed that the effect degree was 3 , as calculated using the mean and standard deviation difference in this group were 0.06 and 0.02 , respectively. Further, the input parameters were set as alpha error $=0.05$ and sample size $=12$, resulting in a power $(1-$ beta error probability) of 1 .

\section{DISCUSSION}

The present study aimed to investigate whether crTMS and nGVS modulate the excitability of vestibulospinal function. An indicator of vestibulospinal response excitability, the H-reflex ratio was not significantly changed by real- or shamcrTMS (first analysis) or by real- or sham-nGVS (second analysis). On the other hand, our third analysis revealed a significant main effect of nGVS on the H-reflex ratio after real-crTMS. These findings indicate that crTMS alone and nGVS alone cannot affect excitability of the vestibulospinal 
TABLE 4 | ANOVA.

\begin{tabular}{|c|c|c|c|c|c|c|c|c|}
\hline & Cases & Sum of squares & df & Mean square & $\mathbf{F}$ & $\mathbf{p}$ & VS-MPR* & $\eta^{2}$ \\
\hline \multirow[t]{4}{*}{ Analysis 1} & CS & 0.049 & 1 & 0.049 & 1.127 & 0.291 & 1.024 & 0.01 \\
\hline & Time & 0.091 & 1 & 0.091 & 2.111 & 0.149 & 1.297 & 0.018 \\
\hline & CS * Time & $4.388 e-4$ & 1 & $4.388 e-4$ & 0.01 & 0.92 & 1 & 0 \\
\hline & Residual & 4.911 & 114 & 0.043 & & & & \\
\hline \multirow[t]{4}{*}{ Analysis 2} & CS & 0.14 & 1 & 0.14 & 3.093 & 0.084 & 1.769 & 0.048 \\
\hline & Time & $5.538 e-5$ & 1 & $5.538 e-5$ & 0.001 & 0.972 & 1 & 0 \\
\hline & CS * Time & 0.134 & 1 & 0.134 & 2.953 & 0.091 & 1.686 & 0.046 \\
\hline & Residual & 2.634 & 58 & 0.045 & & & & \\
\hline \multirow[t]{4}{*}{ Analysis 3} & CS & 0.046 & 1 & 0.046 & 1.85 & 0.18 & 1.193 & 0.031 \\
\hline & Time & 0.136 & 1 & 0.136 & 5.489 & 0.023 & 4.241 & 0.092 \\
\hline & CS * Time & $2.745 e-4$ & 1 & $2.745 e-4$ & 0.011 & 0.917 & 1 & 0 \\
\hline & Residual & 1.29 & 52 & 0.025 & & & & \\
\hline
\end{tabular}

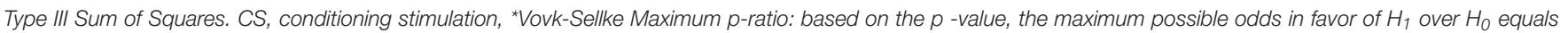
$1 /[-e p \log (p)]$ for $p=0.37$ (Sellke et al., 2001). Bolded value indicates significant.

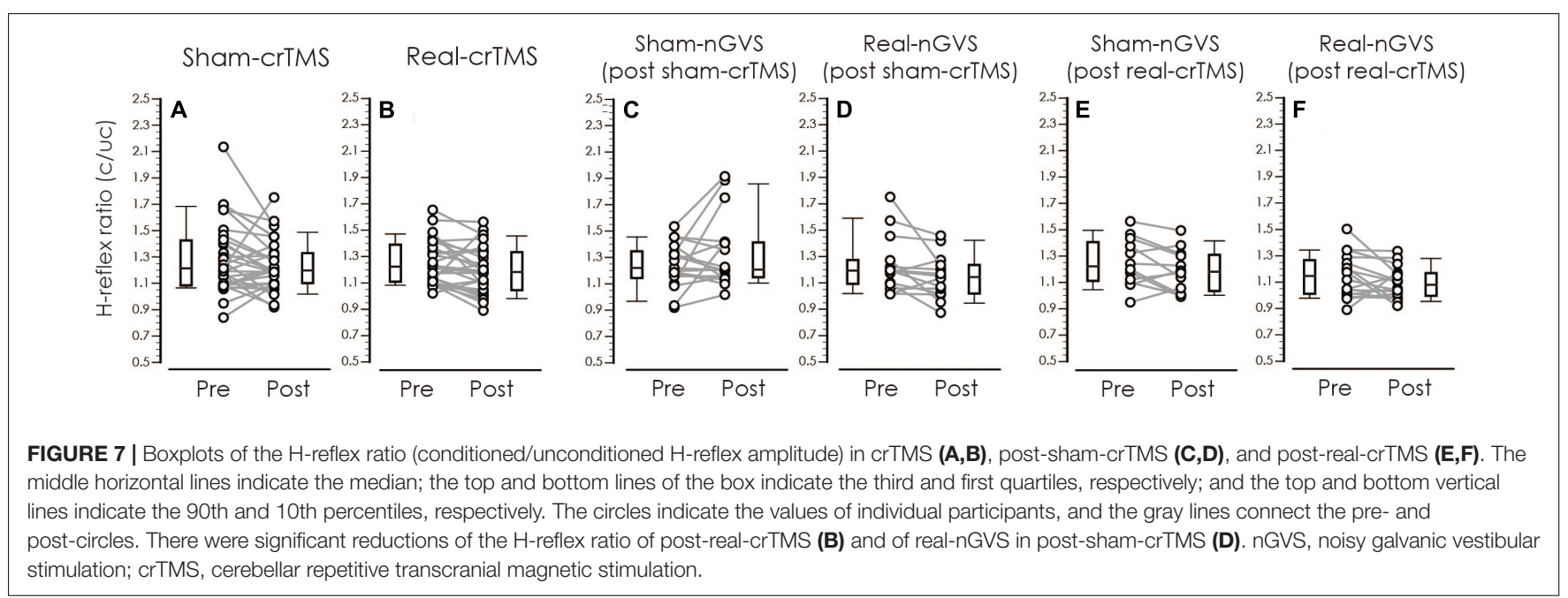

response in participants in the static prone position; however, the nGVS effect could be observed after crTMS. These new findings suggest that crTMS facilitates the effect of nGVS on vestibulospinal function.

Our first analysis revealed that real- and sham-crTMS had no significant effect on the H-reflex ratio, indicating that crTMS does not directly affect excitability of the vestibulospinal response. A previous study showed that low-frequency crTMS reduces CBI, but does not change the excitability of contralateral M1 (Popa et al., 2010), indicating that the low-frequency crTMS disinhibits the excitability of the cerebellar output, but that the stimulation cannot directly affect excitability of remote brain sites. Therefore, in this study, the excitability of vestibular nuclei in the brainstem could not have been directly changed by crTMS alone.

Our secondary analysis showed that the H-reflex ratio was not changed by nGVS after sham-crTMS. This finding suggests the effect of nGVS alone, because sham-crTMS should not affect the cerebellar and vestibular function. Therefore, this result indicates that the application of nGVS alone cannot affect excitability of the vestibulospinal response of a healthy population in the static prone position. Moreover, nGVS modulates the threshold of the motor response through vestibular input and improves body balance in standing humans (Fujimoto et al., 2016, 2018; Wuehr et al., 2017; Inukai et al., 2018). The stochastic resonance and noise addition to non-linear systems inducing the change in plasticity of information processing in neural systems may account for these findings (McDonnell and Ward, 2011). On the other hand, the effect was obtained only in participants with large body sway during upright standing and no effect in participant with small body sway in young adult populations (Inukai et al., 2018). Therefore, in the present study, the effect of nGVS on vestibulospinal function in participants in a static prone position may be small compared to that of participants in an unstable position. As a result, we may have failed to discover an effect of nGVS alone on excitability of the vestibulospinal response in participants in a static prone position.

Our third analysis showed a significant main effect of nGVS on excitability of the vestibulospinal response after real-crTMS. The following mechanisms may account for the facilitation of the nGVS effect after crTMS. The modulation of vestibular reflex is 
affected by the cerebellum (Jang and Kim, 2019). The cerebellum contributes to adaptive changes in the vestibulo-ocular reflex, as shown by the observation that cerebellar lesions disturb long-term adaptive changes in the vestibular reflex (Miles and Eighmy, 1980; Ito, 1998). In a previous study, low-frequency crTMS applied as a pre-conditioning stimulation could not immediately change the vestibulo-ocular movement, but affected the trainability of vestibulo-ocular movement for dynamic gazing (Matsugi et al., 2019). Therefore, in the present study, using the same stimulation paradigm, the change of cerebellar activity induced by crTMS may affect the susceptibility of vestibulospinal response excitability in response to nGVS.

Balance function was not measured in this study, because body movement may affect H-reflex excitability (Knikou, 2008). Therefore, the effect of crTMS and/or nGVS on balance function in positions such as the standing position should be further investigated. As to the question of whether TMS, applied using a butterfly coil, can induce an electrical field on the cerebellar structure, our simulation using SimNIBS suggests that the electrical field, induced during TMS using the butterfly coil, was localized to the cerebellum. Furthermore, Popa reported that crTMS, performed using a figure-eight coil at an angle of $180^{\circ}$ to the coil surface, could modulate the excitability of cerebellar output measured by CBI (Popa et al., 2010). Considered alongside our results, these findings suggest that crTMS applied with a butterfly coil could stimulate the cerebellum. Nevertheless, it is difficult to fully guarantee that deep cerebellar tissue has been stimulated. Recently it was reported that deep brain TMS using H-coil can stimulate the deep brain areas (Roth et al., 2014; Zibman et al., 2019). Therefore, we should conduct future study using $\mathrm{H}$-coil for more certainly stimulating the deep cerebellar tissue in this study design to make sure that our result is due to cerebellar stimulation. Another consideration was the small sample size in the sham-nGVS in post-real-crTMS group. However, our post hoc power analysis revealed a power value of 1 , which is larger than the 0.8 value of the reference study (Faul et al., 2007); accordingly, this analytic power is sufficient to conduct Wilcoxon signed rank sum tests, even in the smallest group.

\section{CONCLUSION}

Low-frequency crTMS alone and nGVS alone were insufficient to modulate excitability of the vestibulospinal response in a young population in the static prone position. In contrast,

\section{REFERENCES}

Ali, A., Rowen, K., and Iles, J. (2003). Vestibular actions on back and lower limb muscles during postural tasks in man. J. Physiol. 546, 615-624.

Balter, S. G., Stokroos, R. J., Eterman, R. M., Paredis, S. A., Orbons, J., and Kingma, H. (2004). Habituation to galvanic vestibular stimulation. Acta Otolaryngol. 124, 941-945.

Britton, T., Day, B., Brown, P., Rothwell, J., Thompson, P., and Marsden, C. (1993). Postural electromyographic responses in the arm and leg following galvanic vestibular stimulation in man. Exp. Brain Res. 94, $143-151$. an effect of nGVS on vestibular function was obtained after crTMS. These findings suggest that the cerebellum modulates vestibulospinal function. Further clinical studies are required to investigate the effect of crTMS and nGVS in patients with vestibulospinal dysfunction.

\section{DATA AVAILABILITY STATEMENT}

The datasets generated for this study are available on request to the corresponding author.

\section{ETHICS STATEMENT}

The studies involving human participants were reviewed and approved by ethics committee of Shijonawate Gakuen University. The patients/participants provided their written informed consent to participate in this study.

\section{AUTHOR CONTRIBUTIONS}

AM, YO, and NY initially designed this study. Experimental equipment for magnetic stimulation was provided by $\mathrm{KH}$ and YS, while AM, NY, and SN provided other equipment. The experiments were conducted by AM, SD, RH, and NM (SD and $\mathrm{RH}$ were blinded assessors). Data analyses were conducted by AM, and NM. AM conducted a simulation of the distribution of E-fields induced by TMS. AM initially wrote the manuscript. All authors revised the manuscript.

\section{FUNDING}

This study was funded by JSPS KAKENHI Grant Number 17K01541.

\section{ACKNOWLEDGMENTS}

We thank all the volunteers for participation in this experiments. We also thank Editage (www.editage.jp) for providing English language editing. This study was partially supported by Japan Agency for Medical Research and Development (AMED).

Crone, C., Hultborn, H., Mazières, L., Morin, C., Nielsen, J., and PierrotDeseilligny, E. (1990). Sensitivity of monosynaptic test reflexes to facilitation and inhibition as a function of the test reflex size: a study in man and the cat. Exp. Brain Res. 81, 35-45.

Daskalakis, Z. J., Paradiso, G. O., Christensen, B. K., Fitzgerald, P. B., Gunraj, C., and Chen, R. (2004). Exploring the connectivity between the cerebellum and motor cortex in humans. J. Physiol. 557, 689-700.

Day, B., Guerraz, M., and Cole, J. (2002). Sensory interactions for human balance control revealed by galvanic vestibular stimulation. Adv. Exp. Med. Biol. 508, $129-137$.

Delfini, C., Diagne, M., Angaut, P., Buisseret, P., and Buisseret-Delmas, C. (2000). Dentatovestibular projections in the rat. Exp. Brain Res. 135, 285-292. 
Faul, F., Erdfelder, E., Lang, A. G., and Buchner, A. (2007). G*Power 3: a flexible statistical power analysis program for the social, behavioral, and biomedical sciences. Behav. Res. Methods 39, 175-191.

Fierro, B., Giglia, G., Palermo, A., Pecoraro, C., Scalia, S., and Brighina, F. (2007). Modulatory effects of $1 \mathrm{~Hz}$ rTMS over the cerebellum on motor cortex excitability. Exp. Brain Res. 176, 440-447.

Fitzpatrick, R., Burke, D., and Gandevia, S. C. (1994). Task-dependent reflex responses and movement illusions evoked by galvanic vestibular stimulation in standing humans. J. Physiol. 478(Pt 2), 363-372.

Fitzpatrick, R., and Day, B. (2004). Probing the human vestibular system with galvanic stimulation. J. Appl. Physiol. 96, 2301-2316.

Fujimoto, C., Egami, N., Kawahara, T., Uemura, Y., Yamamoto, Y., Yamasoba, T., et al. (2018). Noisy galvanic vestibular stimulation sustainably improves posture in bilateral vestibulopathy. Front. Neurol. 9:900. doi: 10.3389/fneur.2018.00900

Fujimoto, C., Yamamoto, Y., Kamogashira, T., Kinoshita, M., Egami, N., Uemura, Y., et al. (2016). Noisy galvanic vestibular stimulation induces a sustained improvement in body balance in elderly adults. Sci. Rep. 6:37575. doi: 10.1038/ srep37575

Ghanim, Z., Lamy, J., Lackmy, A., Achache, V., Roche, N., Pénicaud, A., et al. (2009). Effects of galvanic mastoid stimulation in seated human subjects. J. Appl. Physiol. 106, 893-903. doi: 10.1152/japplphysiol.90594.2008

Goldberg, J., Smith, C., and Fernández, C. (1984). Relation between discharge regularity and responses to externally applied galvanic currents in vestibular nerve afferents of the squirrel monkey. J. Neurophysiol. 51, 1236-1256.

Grimaldi, G., Argyropoulos, G. P., Boehringer, A., Celnik, P., Edwards, M. J., Ferrucci, R., et al. (2014). Non-invasive cerebellar stimulation-a consensus paper. Cerebellum 13, 121-138. doi: 10.1007/s12311-013-0514-7

Haarmeier, T., and Kammer, T. (2010). Effect of TMS on oculomotor behavior but not perceptual stability during smooth pursuit eye movements. Cereb. Cortex 20, 2234-2243. doi: 10.1093/cercor/bhp285

Hardwick, R. M., Lesage, E., and Miall, R. C. (2014). Cerebellar transcranial magnetic stimulation: the role of coil geometry and tissue depth. Brain Stimul. 7, 643-649. doi: 10.1016/j.brs.2014.04.009

Hiraoka, K., Horino, K., Yagura, A., and Matsugi, A. (2010). Cerebellar TMS evokes a long latency motor response in the hand during a visually guided manual tracking task. Cerebellum 9, 454-460. doi: 10.1007/s12311-010-0187-4

Huerta, P. T., and Volpe, B. T. (2009). Transcranial magnetic stimulation, synaptic plasticity and network oscillations. J. Neuroeng. Rehabil. 6:7. doi: 10.1186/17430003-6-7

Iles, J., and Pisini, J. (1992). Vestibular-evoked postural reactions in man and modulation of transmission in spinal reflex pathways. J. Physiol. 455, 407-424.

Inukai, Y., Otsuru, N., Masaki, M., Saito, K., Miyaguchi, S., Kojima, S., et al. (2018). Effect of noisy galvanic vestibular stimulation on center of pressure sway of static standing posture. Brain Stimul. 11, 85-93. doi: 10.1016/j.brs.2017.10.007

Ito, M. (1998). Cerebellar learning in the vestibulo-ocular reflex. Trends Cogn. Sci. 2, 313-321.

Ito, M., Udo, M., Mano, N., and Kawai, N. (1970). Synaptic action of the fastigiobulbar impulses upon neurones in the medullary reticular formation and vestibular nuclei. Exp. Brain Res. 11, 29-47.

Jang, D. C., and Kim, S. J. (2019). Plasticity leading to cerebellum-dependent learning: two different regions, two different types. Pflugers Arch. 471, 927-934. doi: 10.1007/s00424-019-02282-3

Jang, S. H., Lee, M. Y., Yeo, S. S., and Kwon, H. G. (2018). Structural neural connectivity of the vestibular nuclei in the human brain: a diffusion tensor imaging study. Neural Regen. Res. 13, 727-730. doi: 10.4103/1673-5374.230304

Jayasekeran, V., Rothwell, J., and Hamdy, S. (2011). Non-invasive magnetic stimulation of the human cerebellum facilitates cortico-bulbar projections in the swallowing motor system. Neurogastroenterol. Motil. 23, 831-e341. doi: 10.1111/j.1365-2982.2011.01747.x

Jenkinson, N., and Miall, R. C. (2010). Disruption of saccadic adaptation with repetitive transcranial magnetic stimulation of the posterior cerebellum in humans. Cerebellum 9, 548-555. doi: 10.1007/s12311-010-0193-6

Kennedy, P. M., and Inglis, J. T. (2001). Modulation of the soleus H-reflex in prone human subjects using galvanic vestibular stimulation. Clin. Neurophysiol. 112, 2159-2163.

Kennedy, P. M., and Inglis, J. T. (2002). Interaction effects of galvanic vestibular stimulation and head position on the soleus $\mathrm{H}$ reflex in humans. Clin. Neurophysiol. 113, 1709-1714.
Kim, J., and Curthoys, I. S. (2004). Responses of primary vestibular neurons to galvanic vestibular stimulation (GVS) in the anaesthetised guinea pig. Brain Res. Bull. 64, 265-271.

Knikou, M. (2008). The H-reflex as a probe: pathways and pitfalls. J. Neurosci. Methods 171, 1-12. doi: 10.1016/j.jneumeth.2008.02.012

Lowrey, C. R., and Bent, L. R. (2009). Modulation of the soleus H-reflex following galvanic vestibular stimulation and cutaneous stimulation in prone human subjects. Muscle Nerve 40, 213-220. doi: 10.1002/mus.21275

Matsugi, A. (2019). Reader response: cerebello-spinal tDCS in ataxia: a randomized, double-blind, sham-controlled, crossover trial. Neurology 92:1121.

Matsugi, A., Douchi, S., Suzuki, K., Oku, K., Mori, N., Tanaka, H., et al. (2020). Cerebellar transcranial magnetic stimulation reduces the silent period on hand muscle electromyography during force control. Brain Sci. 10:63. doi: 10.3390/ brainsci10020063

Matsugi, A., Iwata, Y., Mori, N., Horino, H., and Hiraoka, K. (2013). Long latency electromyographic response induced by transcranial magnetic stimulation over the cerebellum preferentially appears during continuous visually guided manual tracking task. Cerebellum 12, 147-154. doi: 10.1007/s12311-0120402-6

Matsugi, A., Mori, N., Uehara, S., Kamata, N., Oku, K., Mukai, K., et al. (2014). Task dependency of the long-latency facilitatory effect on the soleus H-reflex by cerebellar transcranial magnetic stimulation. Neuroreport 25, 1375-1380. doi: 10.1097/WNR.0000000000000275

Matsugi, A., and Okada, Y. (2017). Cerebellar transcranial static magnetic field stimulation transiently reduces cerebellar brain inhibition. Funct. Neurol. 32, 77-82.

Matsugi, A., and Okada, Y. (2020). Cerebellar transcranial direct current stimulation modulates the effect of cerebellar transcranial magnetic stimulation on the excitability of spinal reflex. Neurosci. Res. 150, 37-43. doi: 10.1016/j. neures.2019.01.012

Matsugi, A., Ueta, Y., Oku, K., Okuno, K., Tamaru, Y., Nomura, S., et al. (2017). Effect of gaze-stabilization exercises on vestibular function during postural control. Neuroreport 28, 439-443. doi: 10.1097/WNR.000000000000 0776

Matsugi, A., Yoshida, N., Nishishita, S., Okada, Y., Mori, N., Oku, K., et al. (2019). Cerebellum-mediated trainability of eye and head movements for dynamic gazing. PLoS One 14:e0224458. doi: 10.1371/journal.pone.0224458

McDonnell, M. D., and Ward, L. M. (2011). The benefits of noise in neural systems: bridging theory and experiment. Nat. Rev. Neurosci. 12, 415-426. doi: 10.1038/ nrn3061

Miall, R. C., and Christensen, L. O. (2004). The effect of rTMS over the cerebellum in normal human volunteers on peg-board movement performance. Neurosci. Lett. 371, 185-189.

Miles, F. A., and Eighmy, B. B. (1980). Long-term adaptive changes in primate vestibuloocular reflex. I. Behavioral observations. J. Neurophysiol. 43, 1406-1425.

Moliadze, V., Atalay, D., Antal, A., and Paulus, W. (2012). Close to threshold transcranial electrical stimulation preferentially activates inhibitory networks before switching to excitation with higher intensities. Brain Stimul. 5, 505-511. doi: 10.1016/j.brs.2011.11.004

Muto, N., Shinomiya, K., Komori, H., Mochida, K., and Furuya, K. (1995). Spinal cord monitoring of the ventral funiculus function. Analysis of spinal field potentials after galvanic vestibular stimulation. Spine 20, 2429-2434.

Nagel, M., and Zangemeister, W. H. (2003). The effect of transcranial magnetic stimulation over the cerebellum on the synkinesis of coordinated eye and head movements. J. Neurol. Sci. 213, 35-45.

Okada, Y., Shiozaki, T., Nakamura, J., Azumi, Y., Inazato, M., Ono, M., et al. (2018). Influence of the intensity of galvanic vestibular stimulation and cutaneous stimulation on the soleus H-reflex in healthy individuals. Neuroreport 29, 1135-1139. doi: 10.1097/WNR.0000000000001086

Popa, T., Russo, M., and Meunier, S. (2010). Long-lasting inhibition of cerebellar output. Brain Stimul. 3, 161-169. doi: 10.1016/j.brs.2009.10.001

Roth, Y., Levkovitz, Y., Pell, G. S., Ankry, M., and Zangen, A. (2014). Safety and characterization of a novel multi-channel TMS stimulator. Brain Stimul. 7, 194-205. doi: 10.1016/j.brs.2013.09.004

Sellke, T., Bayarri, M. J., and Berger, J. O. (2001). Calibration of $\rho$ values for testing precise null hypotheses. Am. Stat. 55:10. doi: 10.1198/000313001300339950 
Straka, H., Zwergal, A., and Cullen, K. E. (2016). Vestibular animal models: contributions to understanding physiology and disease. J. Neurol. 263(Suppl. 1), S10-S23. doi: 10.1007/s00415-015-7909-y

Thielscher, A., Antunes, A., and Saturnino, G. B. (2015). Field modeling for transcranial magnetic stimulation: A useful tool to understand the physiological effects of TMS? Conf. Proc. IEEE Eng. Med. Biol. Soc. 2015, 222-225. doi: 10.1109/EMBC.2015.7318340

Tremblay, S., Austin, D., Hannah, R., and Rothwell, J. C. (2016). Non-invasive brain stimulation as a tool to study cerebellar-M1 interactions in humans. Cerebellum Ataxias 3:19.

Ugawa, Y., Uesaka, Y., Terao, Y., Hanajima, R., and Kanazawa, I. (1995). Magnetic stimulation over the cerebellum in humans. Ann. Neurol. 37, 703-713.

van Dun, K., Bodranghien, F., Manto, M., and Marien, P. (2017). Targeting the cerebellum by noninvasive neurostimulation: a review. Cerebellum 16, 695-741. doi: 10.1007/s12311-016-0840-7

Welgampola, M. S., and Colebatch, J. G. (2001). Vestibulospinal reflexes: quantitative effects of sensory feedback and postural task. Exp. Brain Res. 139, 345-353.

Wuehr, M., Decker, J., and Schniepp, R. (2017). Noisy galvanic vestibular stimulation: an emerging treatment option for bilateral vestibulopathy. J. Neurol. 264, 81-86. doi: 10.1007/s00415-017-8481-4
Zangemeister, W. H., and Nagel, M. (2001). Transcranial magnetic stimulation over the cerebellum delays predictive head movements in the coordination of gaze. Acta Otolaryngol. Suppl. 545, $140-144$.

Zibman, S., Pell, G. S., Barnea-Ygael, N., Roth, Y., and Zangen, A. (2019). Application of transcranial magnetic stimulation for major depression: coil design and neuroanatomical variability considerations. Eur. Neuropsychopharmacol. doi: 10.1016/j.euroneuro.2019.06.009 [Epub ahead of print].

Conflict of Interest: The authors declare that the research was conducted in the absence of any commercial or financial relationships that could be construed as a potential conflict of interest.

Copyright (c) 2020 Matsugi, Douchi, Hasada, Mori, Okada, Yoshida, Nishishita, Hosomi and Saitoh. This is an open-access article distributed under the terms of the Creative Commons Attribution License (CC BY). The use, distribution or reproduction in other forums is permitted, provided the original author(s) and the copyright owner(s) are credited and that the original publication in this journal is cited, in accordance with accepted academic practice. No use, distribution or reproduction is permitted which does not comply with these terms. 